\title{
Increased concentrations of soluble B7-H3 and interleukin 36 in bronchoalveolar lavage fluid of Children with Mycoplasma pneumoniae pneumonia
}

Zhengrong Chen ${ }^{1 \dagger}$, Xin Zhao ${ }^{2 \dagger}$, Xinxing Zhang ${ }^{1}$, Guangbo Zhang ${ }^{3}$, Huiming Sun ${ }^{1}$, Wujun Jiang ${ }^{1}$, Yuqing Wang ${ }^{1}$, Canhong Zhu', Wei $\mathrm{ji}^{i^{*}}$ and Yongdong Yan $^{1^{*}}$

\begin{abstract}
Background: The purpose of this study is to explore the correlations of interleukin 36 (IL-36) and Soluble B7-H3 (sB7-H3) levels in bronchoalveolar lavage fluid (BALF) with clinical characteristics and laboratory findings.

Methods: A total of 35 children with M. pneumnoiae pneumonia (MPP) and 15 control subjects were enrolled. BALF concentrations of sB7-H3 and IL-36 were detected using enzyme-linked immunosorbent assays and clinical profiles of children with MPP were obtained.

Results: Children with MPP had significantly higher levels of sB7-H3 and IL-36 compared to control subjects (both $P<0.05$ ). Meanwhile, children with pleural effusion had significantly higher levels of sB7-H3 and IL-36 compared to children without pleural effusion (both $P<0.05$ ). BALF concentration of sB7-H3 was strongly associated with concentration of IL-36 $(r=0.796, P<0.0001)$ and sB7-H3 was correlated with duration of fever $(r=0.427, P=0.11)$ and length of stay $(r=0.345, P=0.043)$. Both concentrations of sB7-H3 and IL-36 were significantly decreased in convalescent phase after treatment (both $P<0.05$ ).
\end{abstract}

Conclusion: Both soluble B7-H3 and IL-36 may play an important role in pathogenesis of $M$. pneumoniae infection and sB7-H3 could be useful as a prognostic predictor or biomarker of MPP.

Keywords: Mycoplasma pneumoniae pneumonia, B7-H3, Interleukin 36, Children, Bronchoalveolar lavage fluid

\section{Background}

Atypical pneumonia caused by Mycoplasma pneumoniae (M. pneumoniae) is a leading cause of mortality among the pediatric age group. M. pneumoniae was found in approximately $40 \%$ of children infected with community acquired penumonia (CAP), among which $18 \%$ of the patients required hospitalization [1]. In the past, M. pneumoniae infections were considered to occur mainly in older children, adolescents, and young adults. However, our recent studies have shown that $M$. pneumoniae has become

\footnotetext{
*Correspondence: szdxjiwei@163.com; yyd3060@163.com

The author of Xin Zhao is the co-first author because of the equal contribution.

${ }^{\dagger}$ Equal contributors

'Department of Respiratory Disease, Children's Hospital of Soochow

University, Soochow University, Suzhou, China

Full list of author information is available at the end of the article
}

an important cause of lower respiratory tract infection (LRTI) [2] or bronchiolitis [3] in infant patients using combined molecular and serologic methods.

M. pneumoniae infection can develop into a severe life-threatening disease such as acute respiratory distress syndrome, necrotizing pneumonitis, and fulminant pneumonia [4-6], although M. pneumoniae infection is usually a self-limited disease. Both pathogen invasion and host immune response play roles in severe $M$. pneumoniae infection. We have reported that increased levels of soluble B7-H3 (sB7-H3) and tumor necrosis factor- $\alpha$ (TNF- $\alpha$ ) in peripheral blood may play an important role in immunopathogenesis of $M$. pneumoniae pneumonia (MPP) [7]. In vitro, M. pneumoniae could induce interleukin $1 \beta$ expression and secretion in human monocytic U937 cell lines [8]. Interleukin 36 (IL-36), another 
member of the IL-1 gene family, including 3 agonistic

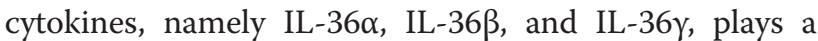
major role in human psoriasis [9] as well as regulating airway inflammation [10] through stimulation on dendritic cells and CD4+ T cells $[11,12]$. IL-36 $\alpha$ can induce neutrophil influx and is also associated with increased mRNA expression of neutrophil-specific chemokines CXCL1 and CXCL2 in the lungs of C57BL/6 [13]. Meanwhile, IL-36 cytokines have a significant effect on the development of Th1 responses [14] which have a potential role in MPP [15], furthermore costimulatory molecule $\mathrm{B} 7-\mathrm{H} 3$ plays a vital role in $\mathrm{T}$ cells differentiation such as Th1 cells [16]. Thus, we presume that $\mathrm{B} 7-\mathrm{H} 3$ might be associated with IL-36 in patients with MPP.

So far, there is no study that reported the expression of IL-36 and sB7-H3 in bronchoalveolar lavage fluid (BALF) of children with MPP. The purpose of this study is to explore the associations between levels of IL-36 and $\mathrm{sB7}-\mathrm{H} 3$ in BALF and clinical characteristics and laboratory findings.

\section{Methods}

\section{Study design}

From Janurary 2014 to December 2014, cases with MPP confirmed by both positive enzyme-linked immunosorbent assay (ELISA) and polymerase chain reaction (PCR) were enrolled. All cases were from 1 month to 14 years old and had fever, cough, tachypnea, chest retractions, abnormal auscultatory findings and radiologic evidence of CAP. Cases were not included if they had chronic lung disease, immunodeficiency, bronchopulmonary malformation, or co-infection. Fiberoptic bronchoscopy was applied if routine treatment of MPP (macrolide $>7$ days and methylprednisolone $>2$ days) was ineffective and patients still have a prolonged fever and radiological deterioration. Finally, BALF samples from 35 children diagnosed with MPP were collected. Radiograph of all patients were conducted 5-7 days after the first bronchoalveolar lavage and second bronchoalveolar lavage was performed if there were no definitive changes between radiographs. Meanwhile, 15 BALF samples of children with foreign body in bronchus were collected as control group. This study was approved by the Institutional Human Ethical Committee of Children's Hospital of Soochow University and the methods were carried out in accordance with the approved guidelines. A written consent was obtained from all the guardians who participated in this study.

\section{Patient evaluation}

The pediatricians completed a questionnaire regarding the demographic and clinical data on hospital admission and discharge. Chest radiographies of all cases were reviewed by the radiologists. BALF samples were collected from all of the enrolled children to detect common etiology using direct immunofluorescence assay (DFA) and PCRs. All the laboratory data was reviewed including blood rutine test, C-reactive protein concentration, immunoglobulin, subpopulation of $\mathrm{T}$ and $\mathrm{B}$ lymphocytes. sB7-H3 and IL-36 concentrations in BALF were detected using ELISA. Of all the cases, convalescent BALFs in 25 children were collected.

\section{Serology of $M$. pneumoniae}

According to the manufacturer's instructions, the specific antibodies against M. pneumoniae (IgG and IgM) were detected in serum samples of children including

Table 1 Demographic and clinical data of children with MPP

\begin{tabular}{|c|c|}
\hline Parameters & Childen with MPP \\
\hline Age (mean $\pm S D$, year) & $5.5 \pm 2.4$ \\
\hline Male (n, \%) & $19(54.3)$ \\
\hline Duration of fever, (25th-75th percentile, d) & $15(13-18)$ \\
\hline Length of stay, (25th-75th percentile, d) & $10(7-13)$ \\
\hline White blood cell counts (mean $\pm \mathrm{SD}, \times 10^{9} / \mathrm{L}$ ) & $9.6 \pm 4.596$ \\
\hline Neutrophils proportion (mean $\pm S D$, \%) & $70.1 \pm 13.8$ \\
\hline C-reactive protein (25th-75th percentile, $\mathrm{mg} / \mathrm{L}$ ) & $25.4(10.0-62.2)$ \\
\hline ALT increase $(n, \%)$ & $8(22.9)$ \\
\hline $\mathrm{LDH}($ mean $\pm \mathrm{SD}, \mathrm{U} / \mathrm{L})$ & $499.0 \pm 171.8$ \\
\hline CKMB (25th-75th percentile, U/L) & $17.5(15-24.4)$ \\
\hline $\lg G($ mean $\pm S D, g / L)$ & $9.8 \pm 4.2$ \\
\hline IgA (25th-75th percentile, g/L) & $1.4 \pm 0.9$ \\
\hline $\operatorname{lgM}($ mean $\pm S D, g / L)$ & $2.3 \pm 1.2$ \\
\hline $\mathrm{CD} 3+\mathrm{T}$ cells (mean $\pm \mathrm{SD}, \%)$ & $67.1 \pm 10.9$ \\
\hline CD3 + CD4+ T cells (mean \pm SD, \%) & $36.6 \pm 9.7$ \\
\hline CD3-CD8+ T cells (mean \pm SD, \%) & $26.2 \pm 6.1$ \\
\hline CD3-CD19+ B cells (mean \pm SD, \%) & $19.2 \pm 8.1$ \\
\hline CD3-CD (16+56+) NK cells (mean \pm SD, \%) & $12.0 \pm 8.1$ \\
\hline CD19+CD23+ B cells (mean \pm SD, \%) & $8.9 \pm 4.7$ \\
\hline \multicolumn{2}{|l|}{ Cytology of BALF } \\
\hline Neutrophils (\%) & 65.6 \\
\hline Lymphocytes (\%) & 7.2 \\
\hline Phagocytes (\%) & 26.4 \\
\hline \multicolumn{2}{|l|}{ Radiologic evaluation (n, \%) } \\
\hline Lobar or segmental opacity & $33(94.3)$ \\
\hline Opacity with pleural effusion & $12(34.3)$ \\
\hline Opacity with pulmonary atelectasis & $3(8.6)$ \\
\hline Macrolide medication $(\mathrm{n}, \%)$ & $35(100)$ \\
\hline Methylprednisolone (n, \%) & $35(100)$ \\
\hline
\end{tabular}

MPP Mycoplasma pneumoniae pneumonia, ALT alanine transarninase, $L D H$ L-lactate dehydrogenase, CKMB MB isoenzyme of creatine kinase, Ig immunoglobulin, $C D$ cluster of differentiation 
acute phase (upon admission) and convalescent phase (upon discharge) using a commercial ELISA kit (Serion ELISA classic M. pneumoniae IgG/IgM, Institute Virion/ Serion, Würzburg, Germany). Acute $M$. pneumoniae infection was defined as either a single positive serum IgM titer (cut-off $13 \mathrm{U} / \mathrm{mL}$ ) or a 4-fold increase in the IgG titer of convalescent serum.

\section{BALF collection and realtime PCR for M. pneumoniae detection}

The procedure of BALF collection using fiber optic bronchoscopy was described previously [17]. First BALF samples were used for $M$. pneumoniae DNA, IL-36 and $\mathrm{sB} 7-\mathrm{H} 3$ detection and other BALF samples were used for cell count after centrifugation at $200 \times \mathrm{g}$ for $10 \mathrm{~min}$ at $4{ }^{\circ} \mathrm{C}$. M. pneumoniae DNA was detected using a real-time PCR commercial kit (Daan Gene Co. Ltd, Guangzhou, China) as described previously [17]. In short, one sample of BALF was shaken for $30 \mathrm{~s}$ and centrifuged at $15,000 \mathrm{~g}$ for $5 \mathrm{~min}$ and the sediment was collected for DNA extraction from a 400- $\mu \mathrm{l}$ sample in accordance with the manufacturer's instructions. PCR amplication was conducted using commercial primers and probes. Quantification curves were plotted using several concentrations of standard control samples.

\section{Multi-pathogen detection}

Other nine viruses were detected using direct immunofluorescence assay or PCRs. Seven common viruses including respiratory syncytial virus, influenza virus types $A$ and $B$, parainfluenza virus types $1-3$, and adenovirus were detected using direct immunofluorescence assay while human metapneumovirus and human bocavirus were detected using PCRs as described previously [18].

\section{Examination of sB7-H3 and IL-36 in BALF}

Collected BALF samples were preserved at $-80{ }^{\circ} \mathrm{C}$ for subsequent assays after routine centrifugation. sB7-H3 ELISA kits were produced by our laboratory as previously described [19] and IL-36 ELISA kits were purchased from R\&D company. All procedures were conducted according to the manufacturer's instructions.

\section{Data analysis}

The Chi-square test or Fisher's exact test were applied if the data was numeral and the Student $t$-test or MannWhitney $U$-test were applied if the data distribution was non-normal. The Pearson or Spearman correlation test was used to assess correlations. A two-sided $p$-value of $<0.05$ was considered statistically significant. All analyses were performed using SPSS for Windows, version 17.0 software (SPSS Inc., Chicago, IL, USA).

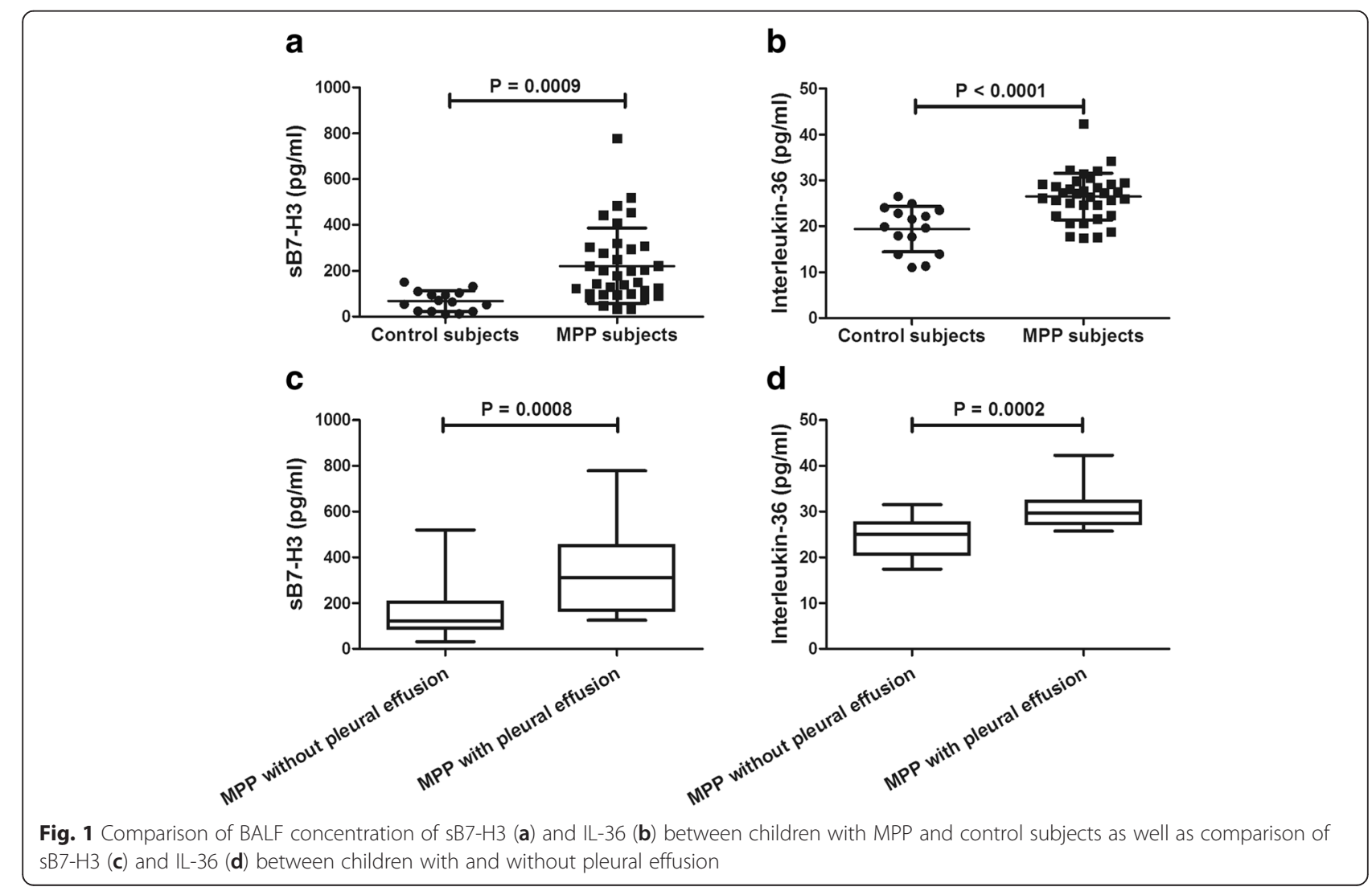




\section{Results}

Demographic and clinical data of children with MPP

The demographic data, clinical characteristics and laboratory findings of children with MPP are shown in Table 1. The average age of control subjects was $4.4 \pm 2.4$ (year), and the male percentage was $60 \%(9 / 15)$. No statistical significance in age and gender was found between children with MPP and control subjects.

BALF concentrations of sB7-H3 and IL-36 in children with MPP and control subjects

As shown in Fig. 1, children with MPP had significantly higher levels of sB7-H3 (221.3 \pm 164.5 vs. $67.6 \pm 45.0, \mathrm{pg} /$ $\mathrm{ml} ; P=0.0009)$ and IL-36 (26.5 \pm 5.2 vs. $19.4 \pm 5.0, \mathrm{pg} /$ $\mathrm{ml} ; P<0.0001)$ compared to control subjects. Among all $M$. pneumoniae infected cases, children with pleural effusion had significantly higher levels of sB7-H3 $(342.6 \pm 186.7$ vs. $158.0 \pm 110.3, \mathrm{pg} / \mathrm{ml} ; P=0.0008)$ and IL-36 (30.6 \pm 4.5 vs. $24.3 \pm 4.1, \mathrm{pg} / \mathrm{ml} ; P=0.002)$ compared to children without pleural effusion.

\section{Correlation between sB7-H3, IL-36 and clinical profiles in children with MPP}

To our interest, BALF concentration of sB7-H3 was strongly associated with concentration of IL-36 as shown in Fig. 2. As shown in Table 2, BALF concentration of sB7-H3 was correlated with duration of fever $(r=0.427$, $P=0.011)$ and length of stay $(r=0.345, P=0.043)$, however no significant correlation was found between IL-36 and clinical parameters.

BALF concentrations of SB7-H3 and IL-36 before and after treatment

All children were treated with Azithromycin $(10 \mathrm{mg} / \mathrm{kg} . \mathrm{d})$ and Methylprednisolone (1-2 mg/kg.d) and no severe complications (bronchiolitis obliterans, bronchiectasis) were found in any case within 6 months follow-up.

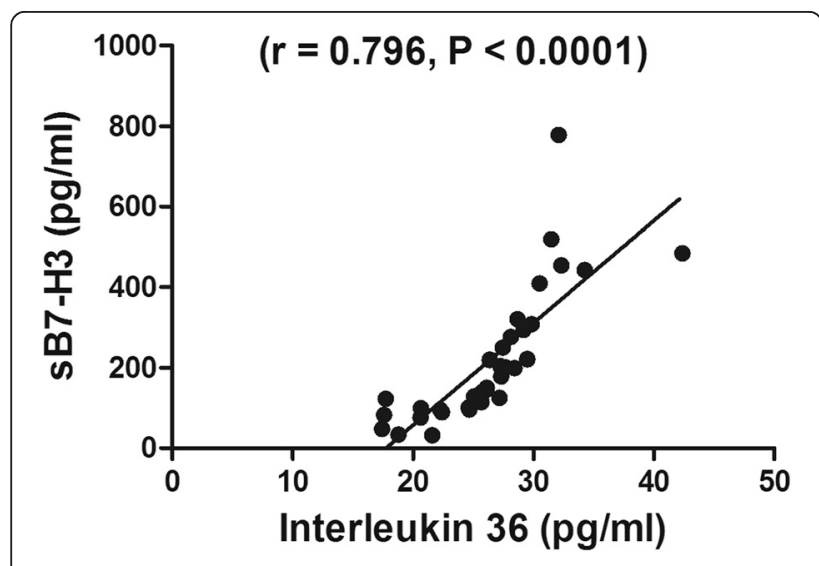

Fig. 2 Correlation between BALF concentration of sB7-H3 and IL-36
Table 2 Correlation between sB7-H3, IL-36 and clinical profiles in children with MPP

\begin{tabular}{|c|c|c|c|c|}
\hline \multirow[t]{2}{*}{ Parameters } & \multicolumn{2}{|l|}{ IL-36 } & \multicolumn{2}{|l|}{ sB7-H3 } \\
\hline & r & $P$ & $r$ & $P$ \\
\hline Duration of fever (d) & 0.144 & 0.409 & 0.427 & 0.011 \\
\hline Length of stay (d) & 0.162 & 0.352 & 0.345 & 0.043 \\
\hline Wite blood cell counts $\left(\times 10^{9} / \mathrm{L}\right)$ & -0.020 & 0.910 & -0.143 & 0.411 \\
\hline Neutrophils percentage (\%) & 0.000 & 0.999 & 0.081 & 0.643 \\
\hline C-reactive protein (mg/L) & 0.100 & 0.566 & 0.113 & 0.518 \\
\hline ALT increase (\%) & -0.169 & 0.332 & -0.185 & 0.187 \\
\hline $\mathrm{LDH}(\mathrm{U} / \mathrm{L})$ & 0.039 & 0.825 & 0.233 & 0.178 \\
\hline CKMB (U/L) & 0.006 & 0.971 & -0.095 & 0.587 \\
\hline $\lg G(g / L)$ & 0.203 & 0.241 & 0.027 & 0.879 \\
\hline $\lg A(g / L)$ & -0.008 & 0.963 & -0.117 & 0.505 \\
\hline $\lg M(g / L)$ & 0.080 & 0.649 & 0.068 & 0.696 \\
\hline CD3 + T cells $(\%)$ & -0.092 & 0.597 & -0.184 & 0.289 \\
\hline CD3 + CD4+ T cells (\%) & -0.050 & 0.774 & 0.004 & 0.982 \\
\hline CD3-CD8+ T cells (\%) & -0.023 & 0.897 & -0.141 & 0.421 \\
\hline CD3-CD19+ B cells (\%) & -0.042 & 0.809 & -0.041 & 0.815 \\
\hline CD3-CD(16 + 56+)NK cells (\%) & 0.167 & 0.337 & 0.227 & 0.190 \\
\hline CD19 + CD23+ B cells (\%) & -0.163 & 0.349 & -0.173 & 0.321 \\
\hline \multicolumn{5}{|l|}{ Cytology of BALF } \\
\hline Neutrophils (\%) & -0.221 & 0.201 & -0.333 & 0.050 \\
\hline Lymphocytes (\%) & 0.089 & 0.610 & 0.048 & 0.786 \\
\hline Phagocytes (\%) & 0.169 & 0.331 & 0.294 & 0.087 \\
\hline
\end{tabular}

MPP Mycoplasma pneumoniae pneumonia, $A L T$ alanine transarninase, $L D H$ L-lactate dehydrogenase, CKMB MB isoenzyme of creatine kinase; Ig: immunoglobulin, $C D$ cluster of differentiation. $P<0.05$ was considered statistically significant

Convalescent BALF samples were obtained from 25 children with MPP. Both concentrations of sB7-H3 and IL-36 significantly decreased in convalescent phase as shown in Fig. 3.

\section{Discussion}

This study describes the expression of sB7-H3 and IL-36 in BALF samples of children with MPP and explores the correlations with clinical profiles. Our study shows that both BALF concentrations of sB7-H3 and IL-36 in children with MPP were significantly increased compared to control subjects and strong correlation was found between $\mathrm{sB7}-\mathrm{H} 3$ and IL-36. In addition, children with pleural effusion had significantly higher concentrations of sB7-H3 and IL-36. Furthermore, BALF concentration of sB7-H3 was associated to duration of fever and hospital stay. We presume that sB7-H3 may be an important prognostic indicator to evaluate the disease severity. 


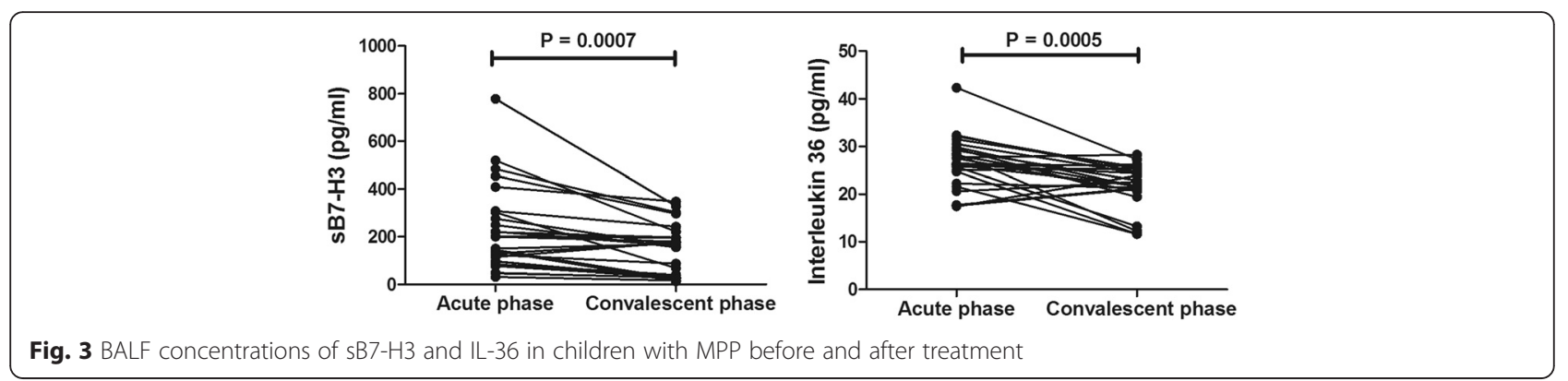

Generally speaking, host immune response caused by M. pneumoniae is a double-edged sword. On one hand, host immune response developed by $M$. pneumoniae infection plays a role in protection from disease deterioration. On the other hand, it could lead to excessive inflammatory response in some cases and consequently developed into severe $M$. pneumoniae infection especially in older children. A wide range of cytokines and chemokines were generated in the respiratory tracts infected by M. pneumoniae, including TNF- $\alpha$, IFN- $\gamma$, IL- 6 , IL-8, IL-17, IL-18 [20-22] and then caused inflammatory infiltration by neutrophils and lymphocytes [23]. In present study, pleural effusion was a sign of excessive inflammation indirectly induced by $M$. pneumoniae infection because no evidence of $M$. pneumoniae in pleural effusions from 3 childen was found using PCR method (data not shown).

B7-H3, a co-stimulatory molecule, plays an important role in the regulation of both innate and Ag-specific $\mathrm{T}$ cell-mediated immune responses and inflammation $[16,24,25]$. In our previous study, B7-H3 could be induced by lipoproteins and functioned as a co-stimulator of innate immunity by augmenting pro-inflammatory cytokine (TNF- $\alpha$, IL-6) release from bacterial cell wall product-stimulated monocytes/macrophages [25]. In a murine model of pneumococcal meningitis, $\mathrm{B} 7-\mathrm{H} 3$ could augment pro-inflammatory cytokine and chemokine production, upregulate NF-kB p65 and MAPK p38 phosphorylation, and enhance the nuclear transactivation of NF- $\mathrm{kB}$ p65 through TLR2-dependent mechanism [26]. Lipoproteins derived from $M$. pneumoniae could induce infiltration of leukocyte cells and production of chemokines and cytokines in BALF [27]. In a word, B7-H3 takes part in inflammatory pathogenesis of MPP and is a good prognostic indicator.

IL-36, a cytokine of IL-1 family member, could express both in epithelia and in immune cells [28]. A recent study [14] demonstrated that IL-36 stimulates the activation of naive $\mathrm{CD} 4+\mathrm{T}$ cell proliferation and IL-2 production. Meanwhile, IL-36 signaling was also critical for Th1-protective immune responses in an experimental model of Bacillus Calmette-Guerin infection [14].
Consequently, IL-36 may act as a signal to activate cells of the innate and adaptive immunity such as DCs and naive $\mathrm{CD} 4+\mathrm{T}$ cells to stimulate host responses against pathogens. This is the first time the high expression of IL-36 in children with MPP and the strong correlation with sB7-H3 were reported. All things considered, we presume that both $\mathrm{B} 7-\mathrm{H} 3$ and IL-36 are crucial in immunopathogenesis of $M$. pneumoniae infection and further studies should be done to elucidate the specific mechanism.

\section{Conclusions}

To summarize, these data support that sB7-H3 and IL-36 may play important roles in inflammatory pathogenesis of $M$. pneumoniae infection. sB7-H3 could be a useful prognostic predictor or biomarker of MPP.

\section{Ethics and consent to participate}

This study was approved by the Institutional Human Ethical Committee of Children's Hospital of Soochow University and the methods were carried out in accordance with the approved guidelines. A written consent was obtained from all the guardians who participated in this study.

\section{Consent to publish}

Not applicable.

\section{Availability of data and materials}

The datasets supporting the conclusions of this article are included within the article and its Additional file 1.

\section{Additional file}

Additional file 1: Supporting data. (XLS $58 \mathrm{~kb})$

Abbreviations

BALF: bronchoalveolar lavage fluid; CAP: community acquired penumonia; ELISA: enzyme linked immunosorbent assay; IL-36: interleukin 36; LRTI: lower respiratory tract infection; $M$. pneumoniae: Mycoplasma pneumoniae; MPP: Mycoplasma pneumoniae pneumnia; PCR: polymerase chain reaction; sB7-H3: Soluble B7-H3; TNF-a: tumor necrosis factor-a. 


\section{Competing interests}

The author(s) declared no potential conflicts of interest with respect to the authorship and/or publication of this article.

\section{Authors' contributions}

Zhengrong Chen, Xinxing Zhang and Wei Ji have made substantial contributions for the concept and design of the main manuscript text; Xin Zhao and Guangbo Zhang have been involved in sB7-H3 and IL-36 detection; Yuqing Wang, Yongdong Yan and Canhong Zhu collected clinical and laboratory data; Huiming Sun and Wujun Jiang analyzed and interpreted the data. All authors have read and approve of the final version of the manuscript.

\section{Acknowledgement}

The authors thank Dr Natalia Tana for her help in revising and editing the manuscript.

\section{Funding}

This work was supported by National Natural Science Foundation of China [Zhengrong Chen, grant number 81401296; Wei Ji, grant number 81570016], Suzhou Science and Technology Projects [Zhengrong Chen, grant number SYS201350; Yongdong Yan, grant number SYS201435], Science and Technology Projects of Jiangsu Provincial Commission of Health and Family Planning [Zhengrong Chen, Q201403], Science and Technology Projects of Chinese Traditional Medicine Bureau of Jiangsu Province[Zhengrong Chen, grant number YB2015176], and Science and Technology Projects of Suzhou sanitary bureau [Yongdong Yan, grant number LCZX201409]. All the funding bodies take part in the design of the study, samples collection, interpretation of data, and writing of this manuscript.

\section{Author details}

${ }^{1}$ Department of Respiratory Disease, Children's Hospital of Soochow University, Soochow University, Suzhou, China. ${ }^{2}$ General surgery department, The First Affiliated Hospital of Soochow University, Soochow University, Suzhou, China. ${ }^{3}$ Clinical Immunology Laboratory, The First Affiliated Hospital of Soochow University, Soochow University, Suzhou, China.

\section{Received: 20 November 2015 Accepted: 10 May 2016 \\ Published online: 17 May 2016}

\section{References}

1. Waites KB, Talkington DF. Mycoplasma pneumoniae and its role as a human pathogen. Clin Microbiol Rev. 2004;17:697-728

2. Wang Y, Hao C, Ji W, Yan Y, Shao X, Xu J. Bronchiolitis associated with Mycoplasma pneumoniae in infants in Suzhou China between 2010 and 2012. Sci Rep. 2015;5:7846.

3. Sun $H$, Chen Z, Yan Y, Huang L, Wang M, Ji W. Epidemiology and clinical profiles of Mycoplasma pneumoniae infection in hospitalized infants younger than one year. Respir Med. 2015;109:751-7.

4. Sztrymf B, Jacobs F, Fichet J, Hamzaoui O, Prat D, Avenel A, et al. Mycoplasma related pneumonia: a rare cause of acute respiratory distress syndrome (ARDS) and of potential antibiotic resistance. Rev Mal Respir. 2013:30:77-80.

5. Wang RS, Wang SY, Hsieh KS, Chiou YH, Huang IF, Cheng MF, et al. Necrotizing pneumonitis caused by Mycoplasma pneumoniae in pediatric patients. Pediatr Infect Dis J. 2004;23:564-7.

6. Shen Y, Zhang J, Hu Y, Shen K. Combination therapy with immunemodulators and moxifloxacin on fulminant macrolide-resistant Mycoplasma pneumoniae infection: a case report. Pediatr Pulmonol. 2013:48:519-22.

7. Chen ZR, Zhang GB, Wang YQ, Yan YD, Zhou WF, Zhu CH, et al. Soluble B7-H3 elevations in hospitalized children with Mycoplasma pneumoniae pneumonia. Diagn Microbiol Infect Dis. 2013;77:362-6.

8. $\quad$ Yang J, Hooper WC, Phillips DJ, Talkington DF. Interleukin-1 beta responses to Mycoplasma pneumoniae infection are cell-type specific. Microb Pathog. 2003:34:17-25.

9. Johnston A, Xing X, Guzman AM, Riblett M, Loyd CM, Ward NL, et al. IL-1 F5, -F6, -F8, and -F9: a novel IL-1 family signaling system that is active in psoriasis and promotes keratinocyte antimicrobial peptide expression. J Immunol. 2011;186:2613-22.

10. Gresnigt MS, van de Veerdonk FL. Biology of IL-36 cytokines and their role in disease. Semin Immunol. 2013;25:458-65.
11. Mutamba S, Allison A, Mahida Y, Barrow P, Foster N. Expression of IL-1Rrp2 by human myelomonocytic cells is unique to DCs and facilitates DC maturation by IL-1F8 and IL-1F9. Eur J Immunol. 2012;42:607-17.

12. Vigne S, Palmer G, Lamacchia C, Martin P, Talabot-Ayer D, Rodriguez E, et al. IL-36R ligands are potent regulators of dendritic and T cells. Blood. 2011;118:5813-23.

13. Ramadas RA, Ewart SL, Iwakura Y, Medoff BD, LeVine AM. IL-36a exerts pro-inflammatory effects in the lungs of mice. PLoS One. 2012;7:e45784

14. Vigne S, Palmer G, Martin P, Lamacchia C, Strebel D, Rodriguez E, et al. IL-36 signaling amplifies Th1 responses by enhancing prolifera-tion and Th1 polarization of naive CD4+ T cells. Blood. 2012:120:3478-87.

15. Tanaka H, Narita M, Teramoto S, Saikai T, Oashi K, Igarashi T, et al. Role of interleukin-18 and T-helper type 1 cytokines in the development of Mycoplasma pneumoniae pneumonia in adults. Chest. 2002;121:1493-7.

16. Chapoval Al, Ni J, Lau JS, Wilcox RA, Flies DB, Liu D, et al. B7-H3: a costimulatory molecule for $\mathrm{T}$ cell activation and IFN-gamma production. Nat Immunol. 2001:2:269-74.

17. Xu D, Li S, Chen Z, Du L. Detection of Mycoplasma pneumoniae in different respiratory specimens. Eur J Pediatr. 2011;170:851-8.

18. Chen ZR, Mize M, Wang YQ, Yan YD, Zhu CH, Wang Y, et al. Clinical and epidemiological profiles of lower respiratory tract infection in hospitalized children due to human bocavirus in a subtropical area of China. J Med Virol. 2014;86:2154-62.

19. Zhang G, Hou J, Shi J, Yu G, Lu B, Zhang X. Soluble CD276 (B7-H3) is released from monocytes, dendritic cells and activated $T$ cells and is detectable in normal human serum. Immunology. 2008;123:538-46.

20. Hardy RD, Jafri HS, Olsen K, Wordemann M, Hatfield J, Rogers BB, et al. Elevated cytokine and chemokine levels and prolonged pulmonary airflow resistance in a murine Mycoplasma pneumoniae pneumonia model: a microbiologic, histologic, immunologic, and respiratory plethysmographic profile. Infect Immun. 2001;69:3869-76.

21. Kurai D, Nakagaki $K$, Wada H, Saraya T, Kamiya S, Fujioka $Y$, et al. Mycoplasma pneumoniae extract induces an IL-17-associated inflammatory reaction in murine lung: implication for mycoplasmal pneumonia. Inflammation. 2013;36:285-93.

22. Narita M, Tanaka H. Late increase of interleukin-18 levels in blood during Mycoplasma pneumoniae pneumonia. Cytokine. 2012;59:18-9.

23. Waites KB, Balish MF, Atkinson TP. New insights into the pathogenesis and detection of Mycoplasma pneumoniae infections. Future Microbiol. 2008;3:635-48.

24. Luo L, Zhu G, Xu H, Yao S, Zhou G, Zhu Y, et al. B7-H3 Promotes Pathogenesis of Autoimmune Disease and Inflammation by Regulating the Activity of Different T Cell Subsets. PLoS One. 2015:10:e0130126.

25. Zhang G, Wang J, Kelly J, Gu G, Hou J, Zhou Y, et al. B7-H3 augments the inflammatory response and is associated with human sepsis. J Immunol. 2010;185:3677-84

26. Chen X, Quinn EM, Ni H, Wang J, Blankson S, Redmond HP, et al. B7-H3 Participates in the Development of Experimental Pneumococcal Meningitis by Augmentation of the Inflammatory Response via a TLR2-Dependent Mechanism. J Immunol. 2012;189:347-55.

27. Shimizu T, Kida Y, Kuwano K. Mycoplasma pneumoniae-Derived Lipopeptides Induce Acute Inflammatory Responses in the Lungs of Mice. Infect Immun. 2008;76:270-7.

28. Gabay C, Towne JE. Regulation and function of interleukin-36 cytokines in homeostasis and pathological conditions. J Leukoc Biol. 2015:97:645-52. 